\title{
Enseigner la langue française autrement
}

\section{Carmen Soddu Gard}

\section{OpenEdition}

\section{Journals}

Édition électronique

URL : http://journals.openedition.org/esp/503

DOI : $10.4000 /$ esp. 503

ISSN : 2532-0319

\section{Éditeur}

Centre d'Information sur l'Éducation Bilingue et Plurilingue

\section{Édition imprimée}

Date de publication : 1 juin 2015

Pagination : 25-32

ISSN : 1127-266X

\section{Référence électronique}

Carmen Soddu Gard, «Enseigner la langue française autrement », Éducation et sociétés plurilingues [En ligne], 38 | 2015, mis en ligne le 01 mars 2016, consulté le 25 février 2021. URL : http:// journals.openedition.org/esp/503 ; DOI : https://doi.org/10.4000/esp.503 


\section{ENSEIGNER LA LANGUE FRANÇAISE AUTREMENT}

\section{Garmen Soddu GaRD}

Entrare facilmente nella lingua senza pensarci troppo, mettendoci del cuore, con la canzone, il cinema, il lavoro manuale nelle cooperative scolastiche e fare degli scambi di classe. Ecco la mia esperienza nelle classi di prima media in Valle d'Aosta.

Rooted in the language, infusing it with all one's heart through songs, the cinema, and arts and crafts in joint school programs and thanks to student exchanges, such is my experience teaching French to pre-teens in the Aosta Valley Region.

Key-words: alternative didactics, teaching French through songs and films

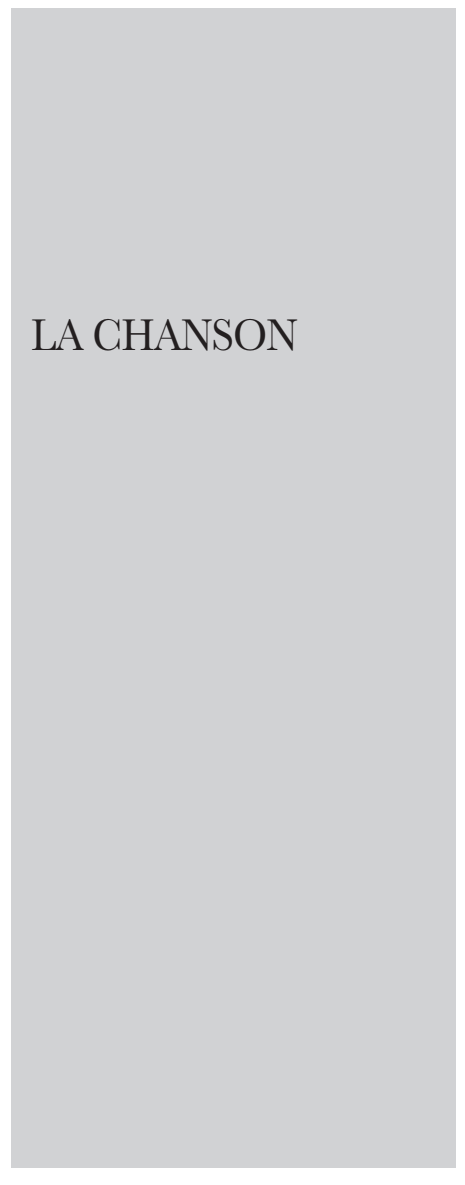

Tout au long de ma carrière d'enseignante de français, j’ai constamment recherché des méthodes d'enseignement et d'apprentissage à la fois pratiques, innovantes, motivantes et stimulantes. Voici donc celles qui m’ont le plus marquée, avec leurs points forts et leurs points faibles et leurs retombées sur les élèves.

Apprendre le Français avec la chanson: tel était l'objectif principal de ce projet qui a caractérisé mes premières années d'enseignement. Il consistait en premier lieu à présenter aux élèves des textes de chansons d'auteurs compositeurs français ou francophones et à les traduire en italien pour en découvrir le lexique. La traduction, bien que son utilité soit très souvent controversée, offre l'avantage "de faire ressortir les connaissances mal intériorisées, de corriger les règles mal acquises et de faire redécouvrir les connaissances perdues" (Bors 1999).

J'ai tout d'abord essayé de capter leur attention en leur annonçant que nous apprendrions des chansons de chanteurs français célèbres. Je leur ai ensuite demandé quelles chansons françaises ils connaissaient. Aucune ! Je leur ai alors demandé quel genre musical ils préféraient afin de leur proposer des chanteurs pouvant leur plaire. Sans trop de surprise de ma part, les élèves me présentèrent une liste de chanteurs et de chansons anglaises ou américaines, confirmant par ce fait le fort recul de la culture francophone dans notre 
Enseigner la langue française autrement

C. SOdDU Gard pays.

J'ai donc dû «choisir pour eux». Il y avait à l'époque une chanson qui passait souvent à la radio et que les jeunes français fredonnaient tout le temps. Il s'agit de «Manhattan-Kaboul» que Renaud et Axelle Red interprétaient en duo. Cette chanson avait été écrite peu après le massacre du onze septembre 2001 et la seconde guerre d'Afghanistan.

Elle traitait des individus anonymes victimes de la guerre et du terrorisme: un jeune portoricain et une jeune afghane parlaient entre eux des atrocités et des victimes innocentes de la guerre.

Ce choix me permettait ainsi une approche et une sensibilisation particulière à la chanson française, celle des faits réels et de la dure actualité.

La chanson est surtout faite pour être chantée. Il n'y a vraiment rien de mieux qu'une bonne chanson pour acquérir la prononciation, l'intonation, le rythme, l'accent d'une langue. Je crois que c'est précisément dans ce domaine que les élèves ont fait d'énormes progrès. Je me rappelle, à ce propos, d'une déclaration d'Adamo, un chanteur belge d'origine italienne. Il voyageait beaucoup et avait confié à une journaliste que, lors de ses tournées en Chine, il chantait en chinois, mais qu'il ne connaissait pas du tout cette langue. Pourtant, en chantant, Adamo donnait vraiment l'impression de pouvoir la parler sans aucune difficulté.

La chanson est aussi une excellente occasion pour remettre à jour notre connaissance du français, avec ses nouvelles tournures, ses expressions idiomatiques en perpétuelle évolution, et pour nous replonger dans la vie et le quotidien de nos cousins.

Souvent considérée comme une activité récréative, parfois superficielle comme genre, elle était mise de côte par certains professeurs. Tout au plus, n'étaient admises dans la salle de classe que certaines chansons poétiques comme celles des auteurs compositeurs classiques tels Brassens, Brel, Leo Ferré ou Barbara.

Pour moi apprendre une langue, c'est fréquenter une autre dimension culturelle et découvrir un univers linguistique différent du nôtre. C'est pourquoi il ne peut se réduire à la simple connaissance des règles de grammaire, aux structures syntaxiques de phrases, ou bien même à l'acquisition du lexique et de notions de civilisation, comme on le fait avec le latin ou le grec classiques. Apprendre une chanson, c'est se plonger dans une langue bien vivante.

Dans le cas de la chanson «Manhattan Kaboul», une des approches utilisées était de faire écouter une première fois la chanson sans le texte, ceci afin d'intéresser la classe et de faire en sorte que tous participent activement en essayant d'en comprendre globalement le 
Enseigner la langue française autrement

C. SOdDU GARD

LES FILMS sens. Lorsqu'un mot ou une phrase n'était pas compris, je demandais de chercher des indices en prêtant attention au rythme, au ton de la voix des chanteurs, à la répétition de mots, et, pour ne pas trop laisser les élèves dans l'incertitude, je fournissais alors quelques renseignements. Je distribuais par la suite le texte et le faisais lire, puis je demandais de réfléchir sur les mots inconnus et de traduire mot après mot, strophe après strophe, ce qui permettait aux élèves de mieux prendre conscience de la spécificité des deux langues aussi bien au plan linguistique que musical. Mais la traduction textuelle débouchait souvent sur des phrases dénuées de sens et obligeait les élèves à rechercher des traductions alternatives, en produisant en eux un important enrichissement linguistique. «Bien qu'elle ait été bannie pendant longtemps de la pédagogie des langues, la traduction est cependant une activité que nous pratiquons tous naturellement. On traduit quand on voit un panneau qui nous interdit de garer notre voiture à l'emplacement visé, ou, quand nous reformulons ce que nous venons de dire si un interlocuteur nous le demande ou enfin quand face à un mot ou expression en langue étrangère, nous cherchons à comprendre la signification de tel ou tel élément de la langue» (Bors 1999).

Une deuxième écoute de la chanson permettait aux élèves de mieux connaitre le texte, qui devenait presque familier (vocabulaire, expressions particulières...), et d'exprimer ce qu'ils avaient ressenti.

Ce qui importait, c'était de voir les élèves participer à une discussion sur le thème évoqué dans la chanson et chanter tous ensemble. Lorsque tout le monde chante d'une même voix, alors même les plus timides se libèrent. Dans ce cas les retombées sont énormes: une plus grande connaissance des thèmes actuels et de la francophonie en général et un patrimoine linguistique enrichi par la découverte du texte, qui pouvait encore être réutilisé dans des jeux de rôle mais aussi comme sujet d'une activité théâtrale ou de débats sur la guerre, ou pour avoir une meilleure connaissance des pays en question (les Etats-Unis et l'Afghanistan).

Les élèves sont de plus en plus liés à l'image, qu'elle provienne d'un ordinateur, du grand ou du petit écran ou de leur portable. C'est une génération de "vidéo jeunes". Avant de montrer un film en entier, je proposais à la classe des bandes annonces afin d'avoir une idée de leurs goûts, à savoir: les genres, les acteurs, les réalisateurs préférés, les chorégraphies. Une fois le film choisi, je proposais une grille de compréhension à remplir: "De quoi s'agit-il? Qui sont les personnages? Où sont-ils? Que font-ils? Quel est le sujet du film? Combien y en a-t-il? Dans la grille ils mettent des observations per- 
Enseigner la langue française autrement

C. SOdDU GARD
LE TRAVAIL MANUEL DANS UNE COOPÉRATIVE SCOLAIRE sonnelles. Enfin je leur faisais exposer les raisons de leurs préférences. La partie la plus intéressante consistait en une activité en tandem, que je pourrais appeler "devinez le film". Il s'agissait de faire parler les élèves à tour de rôle à propos du film sans leur en fournir le moindre détail. C'était aux autres de deviner...

Les films permettaient d'atteindre de nombreux objectifs: approfondir la connaissance de la civilisation (découvrir les lieux, les monuments, les habitudes..), mais aussi de mettre en place différentes activités: débattre et commenter, s'exprimer et donner leur avis sur les nouvelles expressions de la langue.

Un exemple de film proposé aux élèves de première était Hachiko en version française, choisi pour sa forte charge émotionnelle, qui traite d'une profonde amitié entre un professeur de musique et un chien au Tibet.

À la première vision du film, les élèves montrèrent un fort intérêt pour cette histoire émouvante et, même s'ils ne voulaient pas le montrer, beaucoup d'entre eux avaient les larmes aux yeux. Il ne m'était pas possible d'interrompre le film et donc je le montrais jusqu'à la fin. Suivaient quelques questions de compréhension sur le film: (De quoi s'agit-il? Qui sont les principaux protagonistes? Où sommes-nous?) En général, ils répondaient sans difficulté. Je distribuais alors des questionnaires dont les questions respectaient l'ordre des séquences du film: Comment commence l'histoire? Pourquoi le titre «Hachiko»? Que se passe-t-il? Pourquoi le protagoniste prend-il le chien chez lui? Pourquoi ne dit-il pas tout de suite à son épouse qu'il a pris un chien? Quels liens se créent-ils entre eux? Que se passe-t-il ensuite?

Puisque le film marquait émotionnellement les élèves, je ne le passais pas une deuxième fois. J'attendais quelques jours, et je repassais rapidement quelques séquences.

Savoir décrire un animal figure au programme de sixième en Vallée d'Aoste mais aussi en France, ainsi, je m'arrètais sur des expressions concernant le chien: sa race, son origine, ses caractéristiques physiques au début et à la fin, etc.., ensuite je posais des questions sur l'aspect des personnages, le protagoniste, son épouse, le chef de gare, les commerçants et leurs comportements vis-à vis du chien et du protagoniste. Suivaient alors des questions sur les lieux du film: la maison, le jardin, la niche, la gare, etc. Les élèves répondaient avec enthousiasme aux questions et arrivaient à parler du contenu du film et à exprimer leurs sentiments à l'égard de l'animal de compagnie.

La coopérative scolaire était un projet éducatif ayant pour but la création d'objets destinés à la vente et à l'apprentissage d'une langue étrangère, le français dans notre cas. Nous choisîmes le mardi aprèsmidi pour la réalisation de notre travail manuel. Il s'agissait donc de 
Enseigner la langue française autrement

C. SOdDU GARD

\section{DÉMARCHE PÉDAGOGIQUE}

parler en français des objets que nous produisions et qui devaient être vendus à la Foire de St Ours, à l'occasion des fêtes de Pâques ou de Noël. Dans la coopérative scolaire, chaque élève avait un rôle actif et avait par conséquent une motivation supplémentaire pour fréquenter l'école. Le côté pratique, conjugué avec l'apprentissage d'une langue, avait pour effet que même les élèves en difficultés d'apprentissage dans des classes "normales", s'apercevaient qu'lls valaient quelque chose et se montraient enthousiastes même en français. Montrer à un élève que malgré les difficultés il peut réaliser quelque chose de concret dans sa vie, c'est l'objectif de tout enseignant... Pendant mes heures de travail manuel, je parlais, comme d'habitude, en français aux élèves.

D'abord je montrais des techniques de positionnement des mains et des doigts, et au tableau je dessinais le début du travail. Certes, ceux qui avaient quelques notions de couture (enfiler une aiguille et faufiler le tissu) pouvaient à leur tour aider leurs camarades. Tout se faisait en groupe, même ceux qui au début ne savaient pas comment s'y prendre et se piquaient ont fini par s'y mettre. La classe était partagée en groupes de travail: le groupe couture, le groupe aiguilles à tricoter, le groupe crochet et le groupe collage.

On décrivait chaque phase de notre travail, chaque action, nous apprenions des mots nouveaux (moi aussi!). Dans notre atelier, nous produisions des écharpes en laine de toutes les couleurs, des chaussettes en "style autrichien", des bonnets, des sacs, des tricots, etc... Nous coupions du tissu, utilisions des aiguilles pour faufiler les bouts d'étoffe afin de créer des coussins, des maniques pour prendre des plats chauds, des gants de cuisson et bien d'autres objets, après quoi on cousait à la machine pour fignoler "notre création". Le lexique que nous employions était donc celui des points, du crochet, des aiguilles, du passage de l'endroit à l'envers et inversement, etc. Combien de fois ai-je dû répondre et montrer comment s'y prendre! La manualité ce n'est pas du gâteau pour tous! Mais elle est aussi une façon de révéler les aptitudes des élèves, et la démonstration qu'il y a un lien avec le "côté intellectuel" de l'apprentissage: faire un sac en tissu ou au crochet, savoir faire un carré, un rectangle, des écharpes en laines colorées, tout cela suppose des notions à la fois de mathématiques et d'éducation technique...

Le français, durant ces après-midi-là, paraissait plus intéressant pour les élèves car "ils le vivaient". Le français appris dans son contexte naturel est ainsi certainement plus vivant, plus spontané, plus clair et devient pour eux une véritable deuxième langue.

La dernière partie du cours de travail manuel en langue française consistait dans la présentation aux parents des objets qu'ils avaient 
Enseigner la langue française autrement

C. Soddu GARD

\section{LES ÉCHANGES} SCOLAIRES DANS LE CADRE DES PROJETS ALCOTRA créés en leur illustrant les techniques utilisées. L'exposition devait être claire, aisée, articulée et surtout englober le lexique appris.

ALCOTRA (Alpes Latines Coopération TRAnsfrontalière) est un programme de rapprochement européen. La coopération concerne les régions italiennes (Vallée d'Aoste, Piémont, Ligurie) et françaises (Rhône-Alpes, Provence-Alpes-Côtes d'azur).

Son objectif est d'améliorer la qualité de vie des populations et encourager le développement durable des systèmes économiques et territoriaux transfrontaliers grâce à la coopération dans le domaine social, économique, environnemental et culturel. La mise en place d'un partenariat transfrontalier est une condition indispensable pour la présentation de tous les types de projet. Les bénéficiaires peuvent en être des structures publiques ou privées disposant d'une personnalité juridique.

Ces projets avaient pour buts de commémorer le 150ème anniversaire du Rattachement de la Savoie à la France (1860-2010) et l'année suivante (1861-2011) celui du 150ème rattachement de la Vallée d'Aoste à l'Unité Italienne. Certes, c'était une bonne occasion pour les petits Valdôtains de connaître un "ailleurs" et de voir du pays. Aspect qui me paraît important, car, souvent, les élèves n'ont pas la possibilité de se déplacer. Ces échanges donnent cette opportunité à tous.

Lors de ces projets d'échange plusieurs objectifs pédagogiques étaient visés: ancrer les apprentissages en langues vivantes dans une pratique motivante et mettre les élèves en situation de communication en les amenant à développer leurs compétences linguistiques, mais aussi de redonner le goût au savoir et à l'apprentissage surtout pour les élèves en difficulté en intégrant l'outil informatique, valoriser leur activité de production en incitant les élèves transfrontaliers au travail coopératif, s'entraider, faire des recherches de documentation pour les exploiter et enfin les exposer... La réalisation des travaux au sein de l'établissement a permis la création d'un Cdrom sur l'histoire de la langue française dans notre région, de sa défense contre son abolition et de sa «reconnaissance méritée» à travers les lois sur le bilinguisme dans l'école valdôtaine. Les élèves ont réalisé un blog franco-italien (Savoia-Valle d'Aoste) sur les liens historiques des deux régions.

Les retombées linguistiques de ces activités furent importantes car il y a eu cette année-là quatre projets Alcotra.

Les élèves français et valdôtains ont pu découvrir l'histoire du Val d'Aoste et les raisons du particularisme linguistique ainsi que l'histoire du français, du franco-provençal et de l'enseignement bilingue. 
Enseigner la langue française autrement

C. SOddu GARD
Connaître l'histoire linguistique de cette région permet de mieux comprendre pourquoi aujourd'hui encore le français a son importance et est enseigné comme langue en parité avec l'italien. Ainsi de nombreuses conférences étaient organisées avec la présence d'experts linguistiques de part et d'autres des deux régions de montagnes.

Dans cette même optique, l'année suivante, d'autres échanges étaient réalisés afin de mieux connaître les similitudes et les différences entre régions montagnardes. Par le biais des échanges, les élèves communiquaient sur Facebook, MSN, etc. Beaucoup d'entre eux encore aujourd'hui ont gardé un rapport amical et se retrouvent pendant les vacances. L'objectif était toujours de parler un bon français dans un contexte authentique. Connaître le passé pour mieux apprécier le présent!

Pour élaborer ces projets, il fallait d'abord remplir des fiches en y indiquant tous les mouvements relatifs aux transports, aux visites musées, fermes, châteaux, pauses-repas, etc. Cette partie, quoiqu’intéressante, était certainement la plus «ingrate» mais la plus nécessaire car elle permettait aux bureaux administratifs de la Région Vallée d'Aoste de financer tous les déplacements, sans quoi il aurait été impossible de faire quoi que ce soit. A la fin de ces échanges, d'autres fiches-bilan nous ont permis de souligner également les critiques.

Pour ce qui me concerne, de nombreux points forts découlèrent d'une telle expérience d'immersion totale des élèves, accueillis dans les familles, dans un contexte linguistique authentique. Cette expérience se répercutait sur toutes les autres méthodes utilisées dans ma carrière professionnelle par la stimulation d'autres facteurs importants comme l'émotion, la motivation, la sensibilité individuelle, la créativité, etc.

Ce côté affectif n'est pas à dédaigner, bien au contraire: tout commence par une sorte de complicité entre professeur et élèves. Le professeur doit faire preuve de talents très variés (savoir chanter, savoir dessiner, et combien d'autres habiletés, etc.) mais il doit les utiliser non pas en artiste, mais en simple et honnête artisan. Ceci abolit les distances et crée plus facilement un lien avec eux.

Ainsi le Professeur Théodore Froment (Froment 1879) de l'Institut français de l'éducation expliquait dans son livre comment devait être un «enseignant» selon Quintilien, rhéteur et pédagogue latin du $1^{\mathrm{er}}$ siècle après J.G.: «le maître ne doit pas recourir à des punitions pour assurer son autorité: c'est par l' ascendant de son caractère qu'il aura surtout de l'empire sur ses élèves, qu'il prenne avant tout à l'égard de ses élèves les sentiments d'un père (sumat ante omnia parentis 
Enseigner la langue française autrement

C. SOddu GARD

\section{BIBLIOGRAPHIE \\ BORS E. 1999. Traduction en classe de langue, Linguistica (1/2): 238-242. \\ FROMENT T. 1879 Un orateur républicain sous Auguste, Cassius Severus, Annales de la faculté des lettres de Bordeaux, I: 121-138. \\ Savoia-Valle d'Aosta... tra passato e presente. Blog franco-italien sur le webpédagogique.com/3ebilangue/:}

erga discipulos animum); qu'il se regarde comme tenant la place de ceux qui lui ont confié leurs enfants; qu'il n'ait aucun vice et qu'il n'en prenne pas d'autrui; que son austérité n'ait rien de triste, sa douceur rien de relâché: qu'il parle souvent de ce qui est droit et convenable; car plus il donnera de conseils, moins il donnera de punitions. Simple dans son enseignement, exact sans excès, il répondra volontiers aux questions et ira même au devant de ceux qui ne lui en posent pas». Mais si Quintilien exige tant du maitre, il demande en retour à l'élève affection et reconnaissance: «Qu'il aime ceux qui l'instruisent non moins que la science elle-même: qu'il les regarde comme des pères dont il tient non la vie du corps, mais celle de l'esprit (parentes esse non quidem corporum sed mentium credat)».

Il est vrai que ce portrait pourrait sembler peu actuel mais par mon expérience, je peux dire au contraire qu'une bonne partie de ces qualités sont encore valables aujourd'hui.

La fermeté de l'enseignant dans la décision de son projet éducatif doit être évidente. En fait le professeur doit enseigner à apprendre. Il ne suffit pas d'enseigner mais de vouloir faire apprendre. 